\title{
Infección grave por Staphylococcus aureus meticilino sensible productor de leucocidina de Panton-Valentine: reportes de dos casos
} Severe infection by methicillin sensitive Staphylococcus aureus producing
Panton-Valentine leukocidin: reports of two cases

\author{
Dr. Martín Brizuela ${ }^{a}$, Dra. Guadalupe Pérez ${ }^{a}$, Dra. Silvina Ruvinsky ${ }^{a}$ Dra. Claudia Sarkis ${ }^{a}$, \\ Dra. Romina Romero ${ }^{b}$, Bqca. Alejandra Mastroianni ${ }^{b}$, Dra. Lidia Casimir ${ }^{b}$, Bqca. María E. Venuta ${ }^{b}$ \\ Bqca. Verónica Gómez Bonduele y Dra. Rosa Bologna ${ }^{a}$
}

\section{RESUMEN}

Staphylococcus aureus es uno delos principales agentes etiológicos de infecciones en niños provenientes de la comunidad y del ámbito hospitalario. La gravedad de estos cuadros se asocia a factores de virulencia, entre los que se encuentra la leucocidina de Panton-Valentine.

Tanto Staphylococcus aureus resistente como sensible a la meticilina producen esta leucocidina, aunque con frecuencia variable.

Presentamos a dos niños con infección grave por Staphylococcus aureus sensible a la meticilina productor de leucocidina de Panton-Valentine con complicaciones osteoarticulares y endovasculares.

Es fundamental la sospecha diagnóstica, el tratamiento antibiótico adecuadoy el manejo quirúrgico precoz para mejorar el abordaje de estas infecciones.

Se debe mantener la vigilancia epidemiológica para detectar la frecuencia de las infecciones causadas por estas bacterias. Palabras clave: Staphylococcus aureus, leucocidina de PantonValentine, resistencia a la meticilina, enfermedad invasiva.

\section{ABSTRACT}

Staphylococcus aureus is a major etiologic agent of infections in children from the community and the hospital setting. The severity of these conditions is associated with virulence factors, including the Panton-Valentine leukocidin.

Both methicillin resistant and sensitive Staphylococcus aureus produce this leukocidin although with varying frequency.

We present two children with severe infection by sensitive Staphylococcus aureus producer of Panton-Valentine leukocidin with musculoskeletal and endovascular complications.

It is essential the suspected diagnosis, appropriate antibiotic treatment and early surgical management to improve the approach of these infections.

a. Servicio de Control Epidemiológico e Infectología. Hospital de Pediatría "Profesor Dr. Juan P. Garrahan", Ciudad Autónoma de Buenos Aires.

b. Servicio de Microbiología. Hospital de Pediatría "Profesor Dr. Juan P. Garrahan", Ciudad Autónoma de Buenos Aires.

Correspondencia:

Dr. Martín Brizuela, martin.brizuela1984@gmail.com

Financiamiento: Ninguno.

Conflicto de intereses: Ninguno que declarar.

Recibido: 8-10-2015

Aceptado: 23-12-2015
Epidemiological surveillance should be mantained to detect the frequency of infections caused by these bacteria.

Key words:Staphylococcus aureus, Panton-Valentineleukocidin, methicillin resistance, invasive disease.

http:/ /dx.doi.org/10.5546/aap.2016.e237

\section{INTRODUCCIÓN}

Las infecciones por Staphylococcus aureus $\mathrm{S}(\mathrm{a})$ son una importante causa de consulta y morbilidad en la edad pediátrica. ${ }^{1}$

Sa produce toxinas que provocan destrucción celular y daño tisular, enterotoxinas asociadas a intoxicación con alimentos contaminados y exotoxinas relacionadas con el síndrome de shock tóxico estafilocócico. ${ }^{1}$

La leucocidina de Panton-Valentine (PantonValentine leukocidin; PVL, por sus siglas en inglés) es una toxina de $S a$ considerada uno de los factores de virulencia más importantes. ${ }^{2}$ Ha sido relacionada con cuadros de miositis graves y neumonía necrotizante. ${ }^{3,4}$

Estudios de epidemiología molecular realizados en nuestro medio han identificado la PVL en Staphylococcus aureus meticilino resistente (SaMR) y en Staphylococcus aureus meticilino sensible (SaMS). ${ }^{5}$

Se presentan, a continuación, dos niños hospitalizados en el Hospital de Pediatría "Prof. Dr. Juan P. Garrahan" con infecciones graves por SaMS productores de PVL.

\section{Paciente 1}

Niña de 9 años de edad previamente sana y con vacunas completas, que consultó a la Guardia por fiebre e impotencia funcional de la pierna izquierda de 72 horas de evolución. Presentaba el antecedente de traumatismo en el mismo miembro una semana antes.

La paciente se encontraba taquicárdica (155 latidos por minuto) e hipotensa $(95 / 40 \mathrm{mmHg})$ con dificultad respiratoria, por lo que ingresó a la Unidad de Cuidados Intensivos (UCI) con 
diagnóstico de shock séptico secundario a artritis séptica de cadera con distrés respiratorio. Requirió 0,2 gammas $/ \mathrm{kg} /$ minuto de noradrenalina y asistencia respiratoria mecánica (ARM). Luego de la obtención de los hemocultivos, inició un tratamiento con $100 \mathrm{mg} / \mathrm{kg} /$ día de ceftriaxona cada 24 horas, $30 \mathrm{mg} / \mathrm{kg} /$ día de clindamicina cada 8 horas y $60 \mathrm{mg} / \mathrm{kg} /$ día de vancomicina cada 6 horas.

En la ecografía de cadera, se observó la presencia de líquido intraarticular y, en la radiografía de tórax, se observó un infiltrado intersticio-alveolar bilateral con borramiento del seno cardiofrénico izquierdo (Figura 1). Se realizó un drenaje de la cadera izquierda con cultivo del material obtenido.

A las 24 horas de internación, se informaron hemocultivos y líquido articular positivos para SaMS, por lo que se adecuó el tratamiento antibiótico a $150 \mathrm{mg} / \mathrm{kg}$ / día de cefalotina cada 6 horas endovenosa. A los 5 días de tratamiento antibiótico adecuado, persistía con bacteriemia y colección en la cadera izquierda evaluada por ecografía, por lo que se realizó una nueva toilette quirúrgica.

A la semana de internación, se realizó un ecocardiograma, que fue normal y, mediante ecodoppler, se diagnosticó trombosis de la vena ilíaca interna, femoral y safena izquierda.

La paciente requirió ARM durante 8 días e inotrópicos durante 2 días. Permaneció internada en UCI por 20 días y se otorgó el alta médica luego de 55 días de internación.

FIgURA 1. Radiografía de tórax: infiltrado intersticio-alveolar bilateral con borramiento de seno cardiofrénico izquierdo

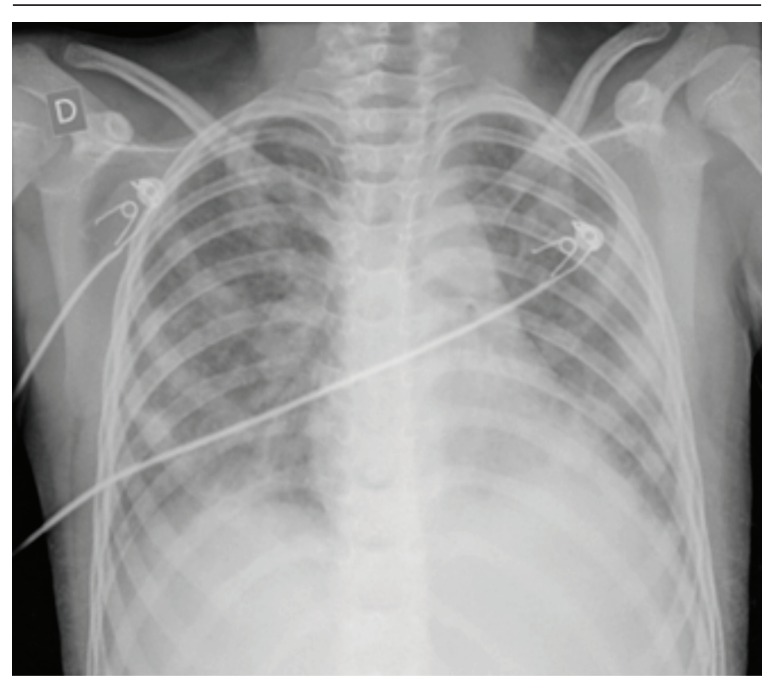

Recibió 6 semanas de tratamiento con cefalotina endovenosa y, posteriormente a cumplir 6 meses de tratamiento oral, con $100 \mathrm{mg} / \mathrm{kg} /$ día de cefalexina cada 6 horas por el diagnóstico de osteomielitis aguda de fémur derecho y neumonía necrotizante por SaMS asociada a foco endovascular.

Tuvo buena evolución clínica durante la internación. A los 2 meses del egreso hospitalario, presentó una fractura patológica del fémur, que requirió inmovilización con yeso pelvipédico durante 6 meses.

\section{Paciente 2}

Varón de 7 años de edad previamente sano, con fiebre de $72 \mathrm{~h}$ de evolución, impotencia funcional y dolor al mover la pierna izquierda.

Al momento del ingreso, se realizó una ecografía de ambas caderas, que mostró líquido en la cadera izquierda y trombosis en la desembocadura de la vena safena izquierda en la vena femoral común izquierda. Se encontraba taquicárdico (155 latidos por minuto) y taquipneico con 40 respiraciones por minuto, por lo que, luego de la toma de hemocultivos, se medicó con $100 \mathrm{mg} / \mathrm{kg} /$ día de ceftriaxona endovenosa cada 24 horas y $60 \mathrm{mg} / \mathrm{kg} /$ día de vancomicina endovenosa cada 6 horas, suponiendo que el cuadro era sepsis de la comunidad con foco osteoarticular y

FIgURA 2. Resonancia magnética nuclear de cadera. Hiperintensidad en metáfisis proximal del fémur izquierdo en $T 1$

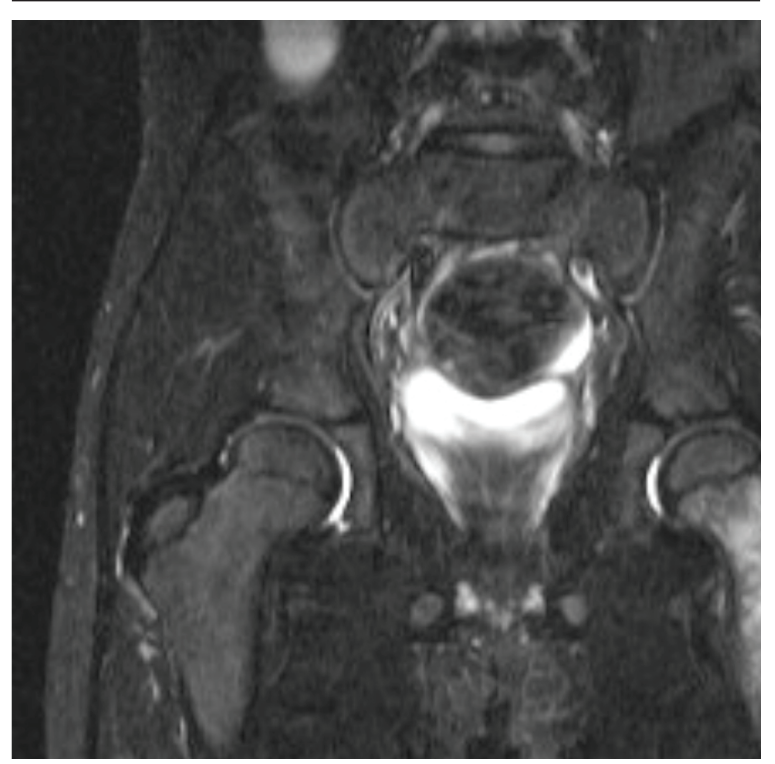


endovascular. Se realizó una artrotomía de cadera y se envió material para cultivo.

A las 48 horas, se identificó SaMS en hemocultivos y líquido articular, por lo que se adecuó el tratamiento con $150 \mathrm{mg} / \mathrm{kg} /$ día de cefalotina cada 6 horas endovenosa.

El paciente persistió con coxalgia luego de una semana de tratamiento antibiótico adecuado y drenaje articular, por lo que se realizó una resonancia magnética nuclear (RMN), que mostró hiperintensidad de la señal en el cuello y metáfisis proximal del fémur izquierdo, con refuerzo con gadolinio compatible con osteomielitis (Figura 2). Se completaron 3 semanas de tratamiento parenteral con cefalotina y, luego, $150 \mathrm{mg} / \mathrm{kg} /$ día de cefalexina cada 6 horas por vía oral durante 6 meses.

No presentó eventos adversos asociados a la medicación y tuvo buena evolución clínica con recuperación de la movilidad del miembro sin secuelas.

En ambos casos, se realizó la reacción en cadena de polimerasa (polymerase chain reaction; PCR, por sus siglas en inglés) en las cepas aisladas en hemocultivos y se identificó la producción de PVL con la presencia de gen LPV y gen 16s DNAr (Figura 3).

FIGURA 3. Reacción en cadena de polimerasa Multiplex Gen $\mathrm{Mec} / \mathrm{Nuc}$. Electroforesis en gel de agarosa al 2,5\% con bromuro de etidio

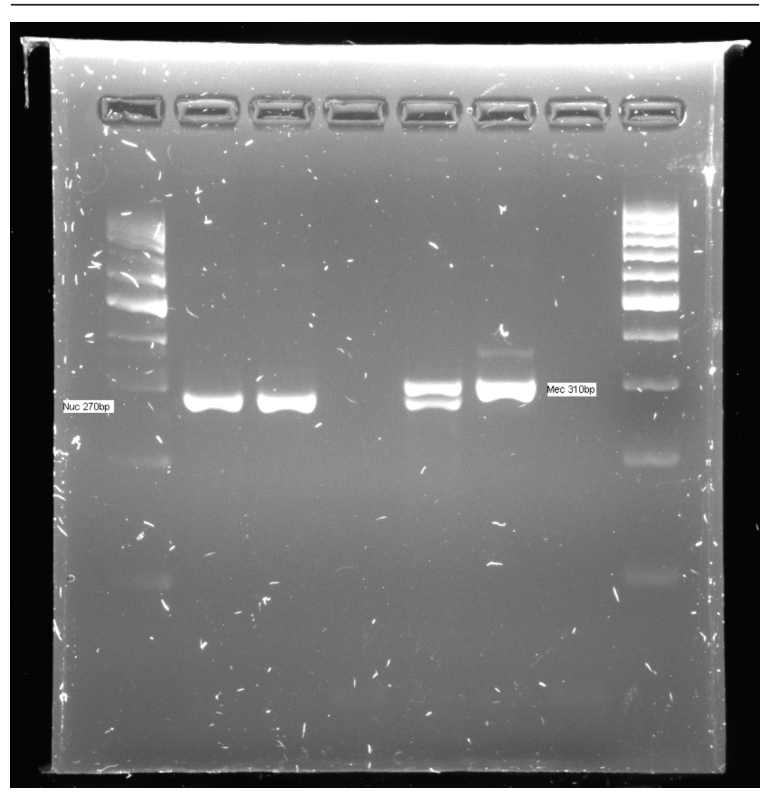

Calle 2 y 3: aislamientos clínicos (SaMS). Calle 4 y 7: control negativo. Calle 5: control positivo (SaMR). Calle 6: SCNMR. Calle 1 y 8: marcador de peso molecular $100 \mathrm{bp}$ SaMS: Staphylococcus aureus meticilino sensible. SaMR: Staphylococcus aureus meticilino resistente. SCNMR: Staphylococcus coagulasa negativo meticilino resistente.

\section{DISCUSIÓN}

$S a$ es uno de los agentes etiológicos involucrados con mayor frecuencia en infecciones de la piel y las partes blandas, osteoarticular, miositis y abscesos en niños provenientes de la comunidad y del ámbito hospitalario a nivel mundial. Estas infecciones, frecuentemente, requieren un tratamiento clínico-quirúrgico, internación prolongada y presentan alta morbimortalidad. 2,6

Con menor frecuencia, produce neumonía necrotizante con empiema pleural, trombosis venosa y cuadros de sepsis o shock séptico secundario, tal como la forma de presentación de nuestros dos pacientes. ${ }^{6}$

La gravedad del cuadro clínico se relaciona con la presencia de distintos factores de virulencia de $S a$, entre los que se destacan las toxinas que producen destrucción tisular, adherencia a los tejidos y colonización. ${ }^{1,7}$

La PVL es una exotoxina involucrada en la destrucción de los leucocitos mediante la formación de poros en la membrana celular. Es transmitida entre cepas por bacteriófagos y codificada por dos genes: lukF PV y lukS PV. A nivel mundial, se describe la producción de PVL por casi todas las cepas de SaMR y, en menor proporción, por SaMS.7

En un estudio realizado en nuestro país en el que predominaron las infecciones graves de la piel y las partes blandas, osteomielitis y sepsis secundaria, se detectó la PVL en el 60\% de los aislamientos de SaMS de la comunidad y del ámbito hospitalario. ${ }^{5}$

Actualmente, existe controversia acerca del rol de la PVL en la gravedad de las infecciones por $S a$.

En los estudios de Lipinska et al. y Kobayashi et al., en los que se inoculó PVL y placebo a conejos, se observó que la reacción inflamatoria producida fue similar en ambos grupos. Los autores concluyeron que múltiples factores diferentes de la PVL estarían asociados a la gravedad de las infecciones producidas por $\mathrm{Sa}^{3,4}$

Wehrhahn et al., observaron que la producción de toxinas diferentes a la PVL se asoció a infecciones de mayor gravedad por $S a$ en adultos. Las infecciones causadas por SaMS fueron más graves que las producidas por SaMR. ${ }^{8}$

Shallcross et al., realizaron una revisión sistemática con estudios que describían la frecuencia de PVL en pacientes con infecciones por $S a$. Concluyeron que las cepas de $S a$ con PVL se encontraron asociadas con más frecuencia a 
infecciones de la piel y las partes blandas, más requerimientos de drenaje quirúrgico y mayor morbilidad. ${ }^{9}$

El inicio del tratamiento antibiótico adecuado y el drenaje quirúrgico precoz de las colecciones y abscesos en pacientes con infecciones graves por $\mathrm{Sa}$ son fundamentales. ${ }^{10}$

Paganini et al., documentaron el predominio de SaMR en las infecciones invasivas por $S a$ en niños en Argentina. ${ }^{11}$ Es conveniente la cobertura antibiótica de SaMR en infecciones producidas probablemente por este microorganismo. Los dos niños presentados recibieron cobertura antibiótica empírica por la sospecha de infección por SaMR.

El antibiótico de elección para el tratamiento de las infecciones producidas por SaMS es la cefalotina por su efecto bactericida mediante el bloqueo de las proteínas de unión a la penicilina (penicillin binding proteins; PBP, por sus siglas en inglés) que participan en la síntesis de la pared celular bacteriana. ${ }^{12}$

La clindamicina actúa inhibiendo la síntesis proteica de $S a$ con probables efectos supresivos en la producción de PVL. ${ }^{13,14}$

Se informa, en la literatura, el uso combinado de clindamicina con antibióticos betalactámicos para disminuir la producción de toxina, con resultados favorables. ${ }^{15}$

En el caso de los pacientes que acá presentamos, se utilizó monoterapia con cefalotina porque la documentación de la producción de PVL fue tardía, independientemente de que ambos pacientes tuvieron buena evolución clínica.

\section{CONCLUSIONES}

Se debe mantener la vigilancia epidemiológica para conocer la sensibilidad antibiótica y la producción de PVL en los clones de $S a$ que predominan a nivel local para ajustar el tratamiento empírico y específico ante este tipo de infecciones de alta morbimortalidad.

\section{REFERENCIAS}

1. Musher DM, Lamm N, Darouiche RO, Young EJ, et al. The current spectrum of Staphylococcus aureus infection in a tertiary carehospital. Medicine (Baltimore) 1994;73(4):186-208.

2. Gillet Y, Issartel B, Vanhems P, FournetJC, etal. Association between Staphylococcus aureus strains carrying gene for
Panton-Valentine leukocidin and highly lethal necrotizing pneumonia in young immunocompetent patients. Lancet 2002;359(9308):753-9.

3. Linpinska U, Hermans K, Meulemans L, Dumitrescu O, et al. Panton-Valentine leukocidin does play a role in the early stage of Staphylococcus aureus skin infections: a rabbit model. PloS One 2011;6(8):e22864.

4. Kobayashi SD, Malachowa N, Whitney AR, Braughton $\mathrm{KR}$, et al. Comparative analysis of USA300 virulence determinants in a rabbit model of skin and soft tissue infection. J Infect Dis 2011;204(6):937-41.

5. Sola C, Saka HA, Vindel A, Bocco JL. High frequency of Panton-Valentineleukocidin genes in invasive methicillinsusceptibleStaphylococcus aureus strains and the relationship with methicillin-resistant Staphylococcus aureus in Córdoba, Argentina. Eur J Clin Microbiol Infect Dis 2007;26(4):281-6.

6. David MZ, Daum RS. Community-associated methicillinresistant Staphylococcus aureus: epidemiology and clinical consequences of an emerging epidemic. Clin Microbiol Rev 2010;23(3):616-87.

7. Cameron DR,Howden BP, Peleg AY. The interface between antibiotic resistance and virulence in Staphylococcus aureus and its impact upon clinical outcomes. Clin Infect Dis 2011;53(6):576-82.

8. Wehrhahn MC, RobinsonJO, Pascoe EM, Coombs GW, et al. Illness severity in community onset invasive Staphylococcus aureus infection and the presence of virulence genes. Jinfect Dis 2012;205(12):1840-8.

9. Shallcross LJ, Fragaszy E, Johnson AM, Hayward AC. The role of the Panton-Valentine leukocidin toxin in staphylococcal disease: a systematic review and metaanalysis. Lancet Infect Dis 2013;13(1):43-54.

10. LiuC,Bayer A, CosgroveSR, Daum RS, etal. Clinical practice guidelines by the Infectious Diseases Society of America for the treatment of methicillin resistant Staphylococcus aureus infections in adults and children. Clin Infect Dis 2011;52(3):e18-55.

11. Paganini H, Della Latta MP, Muller Opet B, Ezcurra G, et al. Estudio multicéntrico sobre las infecciones pediátricas porStaphylococcus aureus meticilino-resistente provenientes de la comunidad en la Argentina. Arch Argent Pediatr 2008;106(5):397-403.

12. Stevens D, Ma Y, Salmi D, McIndoo E, et al. Impact of antibiotics on expression of virulence-associated exotoxin genes in methicillin-sensitive and methicillin-resistant Staphylococcus aureus. J Infect Dis 2007;195(2):202-11.

13. Paramythiotou E, Souli M, Galani I, Giamarellou H, et al. Success stories about severe pneumonia caused by PantonValentine leucocidin-producing Staphylococcus aureus. Braz J Infect Dis 2014;18(3):341-5.

14. Micek ST. Alternatives to vancomycin for the treatment of methicillin-resistant Staphylococcus aureus infections. Clin Infect Dis 2007;45 (Suppl 3):S184-90.

15. Obando I, Croche B, Madrid D, Neth Ol. Osteomielitis aguda por Staphylococcus aureus sensible a la meticilina productor de leucocidina de Panton-Valentine asociada a trombosis venosa profunda y embolismos pulmonares sépticos en dos pacientes pediátricos. Enferm Infecc Microbiol Clin 2011;29(7):550-9. 